\title{
State Effect of Traumatic Experience on Personality Structure
}

\author{
Hong-seock Lee ${ }^{1}$, Sang-Kyu Lee ${ }^{1}$, Heung-Pyo Lee ${ }^{2}$ and Yong-Ku Kim ${ }^{3 凶}$ \\ 1Department of Psychiatry, Kangnam Sacred Heart Hospital, Hallym University Medical Center, Seoul, Republic of Korea \\ ${ }^{2}$ Department of Psychology, Daegu Cyber University, Daegu, Republic of Korea \\ ${ }^{3}$ Department of Psychiatry, Korea University College of Medicine, Ansan Hospital, Ansan, Republic of Korea
}

Objective Personality is defined as the trait-like qualities of a person. However, it has been recently suggested that the state effect of a situation leads to changes in scores on personality assessments. We predicted that traumatic experiences would induce changes not only in personality scores but also in the factor structures of personality assessments.

Methods We conducted a cross-sectional, case-controlled study using two data sets: a traumatized adolescent sample ( $\mathrm{n}=71)$ and a non-traumatized adolescent sample $(\mathrm{n}=296)$. Personality factor structures were compared between the two samples using exploratory factor analyses for 25 lower-ordered subscales of the Temperament and Character Inventory (TCI). In the non-traumatized sample, evaluation of the scree plot suggested a five-factor solution supporting TCI's original seven-factor model.

Results The traumatized sample showed a three-factor structure representing a biological factor, a social factor and an existential factor. This decrease in number of personality factors was caused by strengthened correlations among personality subscales related to coping with traumatic situations. Cloninger's psychobiological model of personality (i.e., temperament-character) was adequate in capturing personality traits of non-traumatized adolescents, but the tripartite view of existential psychology (i.e., body-mind-spirit) clearly corresponded to the factor structure of the traumatized adolescents.

Conclusion The three-factor solution of the present traumatized group is consistent with the tripartite model of personality (i.e., body-mind-spirit), while the five-factor solution of the non-traumatized group corresponds to Cloninger's seven-factor model. This is the first study to describe the state effects of traumatic experiences on personality structure.

Psychiatry Investig 2012;9:361-367

Key Words Personality, Temperament, Character, TCI, Trauma, Adolescent.

\section{INTRODUCTION}

A long history of psychiatry suggests that personality is a trait that maintains itself despite experience. However, recent evidence suggests that situations can have state effects on personality. ${ }^{1}$ Contrary to this belief, studies have shown that personality changes with experience and that personality state rather than traits abide by the proposition of psychotherapy theories: personality change does actually occur. ${ }^{2}$

Although experiences can cause changes in personality, such effects can be observed either as a change in score on personality assessments or as a change in personality structure.

Received: November 21, 2011 Revised: March 5, 2012

Accepted: March 27, 2012 Available online: November 14, 2012

$\triangle$ Correspondence: Yong-Ku Kim, MD, PhD

Department of Psychiatry, Korea University College of Medicine, Ansan Hospital, 123 Jeokgeum-ro, Ansan 425-707, Republic of Korea

Tel: +82-31-412-5140, Fax: +82-31-412-5144, E-mail: yongku@korea.ac.kr

(a) This is an Open Access article distributed under the terms of the Creative Commons Attribution Non-Commercial License (http://creativecommons.org/licenses/by$\mathrm{nc} / 3.0$ ) which permits unrestricted non-commercial use, distribution, and reproduction in any medium, provided the original work is properly cited.
Although it has been well established that the scores on personality assessments can be influenced by internal situation (e.g., depression) as well as external situation (e.g., traumatic experience), ${ }^{3}$ structural change in personality associated with an individual's situation has not been systemically studied. Only theoretical, phenomenological and philosophical works have extensively examined structural change in personality due to external events. ${ }^{4}$ Specifically, the structural change associated with trauma has been termed Transformational Coping, ${ }^{5}$ Quantum Change ${ }^{6}$ and Posttraumatic Growth. ${ }^{7}$

Identifying the structure of personality has been one of the most fundamental goals in personality research. Factor analysis may be used to discover the basic structure of the primary independent dimensions. Factor analysis is a mathematical procedure that helps to sort test responses into relatively homogeneous clusters of highly correlated items. In previous studies, severe depression reportedly strengthened the correlation between the harm avoidance (HA) and cooperativeness (CO) dimensions of the Temperament and Character Inventory (TCI) ${ }^{8,9}$ Because the factor structures of those samples 
were not analyzed, the changes in the correlations between TCI dimensions indicated that the structure in severely depressed patients must be different from those in the normal population and mildly depressed patients.

In this study, we hypothesized that the factor structure of personality in a traumatized sample may be uniquely distinguished from that of a non-traumatized sample. The TCI appears to be a particularly relevant tool to assess changes in personality structure due to traumatic experiences because this model defines the hierarchical development of personality as the organization of behavior resulting from individual experience.

Therefore, we compared the factor structures of the TCIs extracted from its 25 lower- ordered personality dimensions between traumatized and non-traumatized adolescents. In this process, we chose to use an exploratory rather than a confirmatory factor analysis and did not separate the 25 subscales into the original temperament and character dimensions because it is not known whether the factor structure of the TCI varies between the two samples.

\section{METHODS}

\section{Subjects}

Two data sets of Korean adolescents were used: a traumatized sample $(\mathrm{n}=71)$ and a non-traumatized sample $(\mathrm{n}=296)$. The traumatized samples consisted of the Incheon Fire Disaster in October 1999. This disaster resulted in the deaths of 54 middle and high school boys and girls and injuries of 80 students. The included student had experienced events such as hearing the screaming of their friends, loss of friends, fear of death, near suffocation, and loss of consciousness, and the participants were homogenous in age, educational level, and residence. The survivors were 46 (65\%) boys and 25 (35\%) girls, with a mean age of 16.2 years $(\mathrm{SD}=1.1)$. The students were taken to the Ansan Hospital of Korea University Medical Center for treatment and evaluation of physical injuries and psychological distress three months after the disaster. The authors conducted diagnostic interviews and gathered detailed information about their traumatic experience and the symptoms from which they suffered. One student was excluded from the traumatized sample due to the presence of a previous mental disorder. Psychological assessment of the traumatized sample was carried. Their mean (SD) Beck Depression Inventory score was 25.9 (11.2), their mean (SD) State-Trait Anxiety Inventory (STAI)-state was 61.0 (9.8), their mean (SD) STAI-trait was 56.2 (9.4), their mean (SD) Impact of Event Scale (IES)-intrusion was 21.5 (9.1), their mean (SD) IES-avoidance was 24.9 (9.0), and their mean (SD) IES-total was 46.0 (16.8).
For the non-traumatized group, a total of 486 high-school students from Incheon City in Korea were recruited between 2002 and 2004 among interested volunteers who self -reported never having been under psychiatric treatment. Subjects who reported a history of trauma as defined by the criteria of DSM-IV, and subjects with a score greater than 1.5 on the Global Symptom Index of the Korean version of the Symptom Check List- $90^{10}$ were excluded. The final non-traumatized sample consisted of 296 healthy Korean individuals (180 boys, 116 girls) who were age and sex-matched to the traumatized students. The mean age of the non-traumatized sample was 16.3 years $(\mathrm{SD}=0.7)$ and $60 \%$ were male. The research ethics committee of Ansan Hospital of Korea University Medical Center approved this study. Written informed consents were obtained from the subjects.

\section{Study tools}

\section{Temperament and character inventory (TCI)}

All subjects completed the Korean version of Cloninger's $\mathrm{TCI},{ }^{11}$ which is a true-false questionnaire that describes seven higher-order and 25 lower-order traits of temperament and character by means of 240 items. The TCI is constructed based on two basic templates of personality: temperament and character. In his psychobiological model, ${ }^{12}$ Cloninger proposed that temperament involves automatic, preconceptual responses to stimuli, presumably reflecting moderately heritable and stable biases throughout life via information processing by the preconceptual memory system. In contrast, character dimensions are related to the conceptual memory system and refer to self-concepts and individual differences in goals and values that are able to mature progressively throughout life. The TCI temperament template consists of four higher-order dimensions that include novelty seeking (NS), harm avoidance (HA), reward dependence (RD), and persistence (P). The TCI character template is described by the three higher-order character dimensions that include cooperativeness (C), self-directedness (SD), and self-transcendence (ST). Each of the seven dimensions is composed of three to five subscales. Thus, the TCI has a total of 25 lower-order subscales: exploratory excitability versus stoic rigidity (NS1), impulsiveness versus reflection (NS2), extravagance versus reserve (NS3), disorderliness versus regimentation (NS4), anticipatory worry versus uninhibited optimism (HA1), fear of uncertainty versus confidence (HA2), shyness with strangers versus gregariousness (HA3), fatigability and asthenia versus vigor (HA4), sentimentality versus insensitivity (RD1), attachment versus detachment (RD3), dependence versus independence (RD4), persistence versus irresoluteness (P), responsibility versus blaming (SD1), purposefulness versus 
lack of goal direction (SD2), resourcefulness (SD3), self-acceptance versus self-striving (SD4), congruent second nature (SD5), social acceptance versus social intolerance (C1), empathy versus social disinterest $(\mathrm{C} 2)$, helpfulness versus unhelpfulness (C3), compassion versus revengefulness (C4), integrated conscience (C5), self-forgetfulness versus self-conscious experience (ST1), transpersonal identification versus self-isolation (ST2), and spiritual acceptance versus rational materialism (ST3).

\section{Statistical analysis}

For each data set, exploratory scale-level factor analyses were carried out on the 25 subscales of TCI using the principal-components method with oblique (Promax) rotation. The internal consistency of Cloninger's original seven dimensions in both groups and that of the factors extracted by exploratory factor analysis were assessed using Cronbach's alpha. Pearson's $\mathrm{r}$ coefficients were calculated to measure correlations among the TCI scales. SPSS for WINDOWS (version 12.0) was used in these analyses.

\section{RESULTS}

Exploratory factor analyses of the 25 primary subscales showed that patterns of salient loadings differed between the two groups, but each solution generally had interpretable and meaningful structure. The number of retrained factors was determined by examining the scree plot of the eigenvalues. Table 1 and 2 show the factor pattern matrix based on PROMAX oblique rotation and factor intercorrelations for the normative sample and the traumatized sample, respectively.

In the non-traumatized group, the evaluation of the scree plot suggested a five-factor solution, accounting for $54.0 \%$ of the total variance. Each of the five factors explained $19.2 \%$, $11.5 \%, 9.9 \%, 7.4 \%$, and $5.9 \%$ of the remaining variance, respectively (Table 1). When three to seven factors were forced, their psychological contents were not interpretable, and the pattern of factor loadings was more complex. Moreover, the eigenvalues of the correlation matrices showed a rapid decrement in explainable variance after extraction of the fifth factor. Rotation of the sixth factor explained only an additional $2.4 \%$ of the variance. Despite the difference in factor numbers from those of Cloninger's expectations, each of the primary subscales loaded onto Cloninger's original seven factors, with the exception of the NS1, P and SD subscales. NS1 and SD showed relatively complex loadings, and P did not load onto a separate factor. Factor I was characterized by salient loadings on all the HA and SD subscales except SD4 (self-acceptance versus self-striving). Accordingly, the factor was labeled harm avoidance and self-directedness. All C subscales and SD4 had the highest loadings on Factor II. Thus, Factor II was labeled cooperativeness. Factor III was identified as self-transcendence and was purely loaded by the ST subscales. All NS subscales and P had the highest loadings on Factor IV, which was labeled novelty seeking. Factor $\mathrm{V}$ was characterized by salient loadings on all RD subscales. Thus, Factor $\mathrm{V}$ was identified as

Table 1. Factor analysis of the TCl primary subscales in the normative sample $(\mathrm{N}=296)$

\begin{tabular}{|c|c|c|c|c|c|}
\hline \multicolumn{6}{|c|}{ Promax factor loadings } \\
\hline Primary & Factor & Factor & Factor & Factor & Factor \\
\hline subscales & 1 & 2 & 3 & 4 & 5 \\
\hline SD3 & 0.79 & & & & \\
\hline HA2 & -0.74 & & & & \\
\hline HA1 & -0.74 & & & & \\
\hline HA3 & -0.60 & & & & \\
\hline HA4 & -0.60 & & & & \\
\hline SD5 & 0.58 & & & -0.47 & \\
\hline SD2 & 0.58 & & & -0.33 & \\
\hline SD1 & 0.48 & 0.41 & -0.37 & & \\
\hline NS1 & 0.47 & & 0.32 & & 0.29 \\
\hline $\mathrm{C} 4$ & & 0.77 & & & \\
\hline $\mathrm{C} 1$ & & 0.75 & & & \\
\hline $\mathrm{C} 3$ & & 0.62 & & & 0.29 \\
\hline SD4 & & 0.56 & & 0.27 & -0.36 \\
\hline C5 & & 0.55 & & -0.28 & \\
\hline C2 & & 0.44 & & & \\
\hline ST1 & & & 0.85 & & \\
\hline ST2 & & & 0.82 & & \\
\hline ST3 & & & 0.79 & & \\
\hline NS2 & & & & 0.77 & \\
\hline NS3 & & & & 0.52 & \\
\hline $\mathrm{P}$ & 0.47 & & & -0.51 & \\
\hline NS4 & & & & 0.44 & \\
\hline RD3 & -0.32 & & -0.27 & & 0.73 \\
\hline RD4 & & & & & 0.65 \\
\hline RD1 & & 0.32 & 0.39 & & 0.41 \\
\hline \multicolumn{6}{|l|}{ Interfactor } \\
\hline F1 & 1.00 & & & & \\
\hline $\mathrm{F} 2$ & 0.15 & 1.00 & & & \\
\hline F3 & 0.29 & -0.07 & 1.00 & & \\
\hline $\mathrm{F} 4$ & -0.03 & -0.05 & 0.02 & 1.00 & \\
\hline F5 & 0.07 & 0.09 & 0.28 & -0.07 & 1.00 \\
\hline $\begin{array}{l}\text { Percentage of } \\
\text { explained variance* }\end{array}$ & 19.2 & 11.5 & 9.9 & 7.4 & 6.0 \\
\hline
\end{tabular}

Factor loadings $<0.25$ have been omitted. *percentage of variance explained by each factor (eliminating other factors). TCI: temperament and character inventory, F1: factor 1, F2: factor 2, F3: factor 3, F4: factor 4, F5: factor 5, NS: novelty seeking, HA: harm avoidance, RD: reward dependence, P: persistence, SD: self-directedness, C: cooperativeness, ST: self-transcendence 
reward dependence.

In contrast, three-factor solution accounted for $67.8 \%$ of the total variance in the traumatized sample (Table 2). The rotated components accounted for $51.5 \%, 9.6 \%$, and $6.7 \%$ of the remaining variance, respectively. All of the $\mathrm{RD}, \mathrm{C}$, and $\mathrm{SD}$ subscales, except for SD4 (self-acceptance), had the highest loadings on Factor I, which was interpreted as a social factor. All NS and HA subscales, except for NS1 (exploratory excit-

Table 2. Factor analysis of the TCl primary subscales in the Traumatized sample $(\mathrm{N}=71)$

\begin{tabular}{|c|c|c|c|}
\hline \multicolumn{4}{|c|}{ Oblique PROMAX factor loadings } \\
\hline $\begin{array}{l}\text { Primary } \\
\text { subscale }\end{array}$ & $\mathrm{F} 1$ & $\mathrm{~F} 2$ & F3 \\
\hline $\mathrm{CO} 1$ & 0.99 & & \\
\hline $\mathrm{CO} 2$ & 0.83 & & \\
\hline SD5 & 0.83 & & \\
\hline SD1 & 0.80 & & \\
\hline $\mathrm{CO} 5$ & 0.78 & & \\
\hline $\mathrm{CO} 4$ & 0.78 & & \\
\hline SD2 & 0.73 & & \\
\hline $\mathrm{CO} 3$ & 0.73 & & \\
\hline RD1 & 0.66 & & \\
\hline SD3 & 0.61 & & \\
\hline $\mathrm{RD} 3$ & 0.61 & & \\
\hline $\mathrm{RD} 4$ & 0.56 & 0.41 & \\
\hline HA3 & & 0.90 & \\
\hline NS2 & & 0.87 & \\
\hline HA4 & & 0.86 & \\
\hline HA1 & & 0.75 & \\
\hline HA2 & & 0.71 & \\
\hline NS3 & & 0.59 & \\
\hline NS4 & & 0.50 & \\
\hline ST1 & & & 0.87 \\
\hline ST3 & & & 0.73 \\
\hline ST2 & & & 0.71 \\
\hline Persistence & & & 0.58 \\
\hline NS1 & & & 0.58 \\
\hline SD4 & & & 0.39 \\
\hline \multicolumn{4}{|l|}{$\begin{array}{l}\text { Interfactor } \\
\text { intercorrelation }\end{array}$} \\
\hline F1 & 1.00 & & \\
\hline $\mathrm{F} 2$ & 0.56 & 1.00 & \\
\hline $\mathrm{F} 3$ & 0.55 & 0.37 & 1.00 \\
\hline $\begin{array}{l}\text { Percentage of } \\
\text { explained variance* }\end{array}$ & $51.5 \%$ & $9.6 \%$ & $6.7 \%$ \\
\hline
\end{tabular}

Factor loadings $<0.25$ have been omitted. *percentage of variance explained by each factor (eliminating other factors). TCI: temperament and character inventory, F1: factor 1, F2: factor 2, F3: factor 3, F4: factor 4, F5: factor 5, NS: novelty seeking, HA: harm avoidance, RD: reward dependence, P: persistence, SD: self-directedness; C: cooperativeness, ST: self-transcendence ability), had the highest loadings on Factor II. Factor II was interpreted as a behavioral activation system and behavioral inhibition system (BAS/BIS) factor. All subscales of the ST, P (RD2-persistence), NS1 (exploratory excitability versus stoic rigidity), and SD4 (self-acceptance versus self-striving) loaded on Factor III, which was identified as the existential factor. The SD4 subscale also showed a high loading on Factor II, but the SD4 subscale was classified on Factor III according to the view that self-acceptance is a prerequisite for self-transcendence. $^{13}$

Pearson intercorrelations between all of the TCI scales were higher in the traumatized group than they were in the nontraumatized group. All seven scales were highly intercorrelated in both groups (Table 3). In the traumatized group, the highest correlations were observed between $\mathrm{CO}$ and $\mathrm{RD}$ (0.87), CO and SD (0.80), RD and SD (0.73), NS and RD (0.76), and NS and HA (0.71). In the non-traumatized group, the correlations greater 0.40 were those of SD with P $(0.41)$, and SD with HA (-0.53). The five factors extracted from the non-traumatized sample were relatively orthogonal, while the three factors of the traumatized sample were relatively oblique (Table 1). In the non-traumatized sample, factor intercorrelations (r) ranged from 0.01 to 0.29 while those among the three factors in the traumatized sample exceeded 0.37.

\section{DISCUSSION}

This is the first study comparing the TCI's factor structure in traumatized and non-traumatized adolescents. Previous personality research with non-traumatized adolescent $s$ has focused on the possibility of the structural reorganization of personality dimensions in response to traumatic experience. We were able to confirm our hypothesis that the factor structure of personality in a traumatized sample may be uniquely distinguished from that of a non-traumatized sample. The five-factor solution in the non-traumatized group was caused by the known instability of $\mathrm{P}$ and the unexpected collapse of HA into SD subscales, which roughly corresponded to Cloninger's original seven-factor model of personality. Additionally, the traumatized group showed a three-factor structure of personality representing biological, social and existential factors. This study also demonstrated that factor analysis can be used to generate hypotheses regarding the causal mechanisms of each factor by understanding the pattern of correlations within a set of observed variables. Thus, it can be posited that the empirical causes of each of the three factors in the traumatized sample were based on the previous results of the personality assessment.

First, the biological factor extracted from the traumatized group was congruent with the two-factor theory of tempera- 
Table 3. Correlations among the $\mathrm{TCl}$ scales in the traumatized and the normative samples

\begin{tabular}{|c|c|c|c|c|c|c|c|c|c|c|c|c|}
\hline & \multicolumn{6}{|c|}{ Traumatized sample } & \multicolumn{6}{|c|}{ Normative sample } \\
\hline & NS & $\mathrm{HA}$ & $\mathrm{RD}$ & $\mathrm{P}$ & $\mathrm{SD}$ & $\mathrm{C}$ & NS & $\mathrm{HA}$ & $\mathrm{RD}$ & $\mathrm{P}$ & SD & $\mathrm{C}$ \\
\hline HA & $0.71^{* *}$ & & & & & & $-0.30^{* *}$ & & & & & \\
\hline $\mathrm{RD}$ & $0.76^{* *}$ & $0.69^{* *}$ & & & & & -0.07 & $0.21^{* *}$ & & & & \\
\hline $\mathrm{P}$ & $0.49^{* *}$ & $0.24^{*}$ & $0.57^{* *}$ & & & & $-0.35^{* *}$ & -0.03 & -0.01 & & & \\
\hline SD & $0.64^{* *}$ & $0.52^{* *}$ & $0.73^{* *}$ & $0.69^{* *}$ & & & $-0.53^{* *}$ & -0.02 & 0.04 & $0.41^{* *}$ & & \\
\hline $\mathrm{C}$ & $0.68^{* *}$ & $0.62^{* *}$ & $0.87^{* *}$ & $0.56^{* *}$ & $0.80^{* *}$ & & -0.12 & -0.03 & $0.34^{* *}$ & 0.10 & $0.32^{* *}$ & \\
\hline ST & $0.61^{* *}$ & $0.45^{* *}$ & $0.58^{* *}$ & $0.60^{* *}$ & $0.58^{* *}$ & $0.56^{* *}$ & -0.19 & $0.24^{* *}$ & 0.17 & $0.17^{*}$ & 0.09 & $0.16^{*}$ \\
\hline
\end{tabular}

${ }^{*}$ correlation is significant at the 0.05 level (two-tailed), ${ }^{* *}$ correlation is significant at the 0.01 level (two-tailed). TCI: temperament and character inventory, F1: factor 1, F2: factor 2, F3: factor 3, F4: factor 4, F5: factor 5, NS: novelty seeking, HA: harm avoidance, RD: reward dependence, P: persistence, SD: self-directedness, C: cooperativeness, ST: self-transcendence

ment, ${ }^{14}$ which posits that general motivational systems consist of a behavioral activation and behavioral inhibition system underlying behavior and affect. The behavioral activation system (BAS) is regarded as being responsible for the experience of positive feelings in response to signals of reward, non-punishment, and escape from punishment ${ }^{15}$ and it is believed that dopaminergic pathways play a central role in the system. ${ }^{16}$ However, the behavioral inhibition system (BIS) is comprised of the septo-hippocampal system, which is responsible for the experience of negative feelings in response to signals of punishment, non-reward, and novelty. ${ }^{15}$ In fact, among Cloninger's temperament dimensions, the NS and HA dimensions correspond precisely with these two fundamental templates of temperament. ${ }^{17} \mathrm{NS}$, proposed to associate with variation in dopamine level, is a tendency toward exploratory activity, intense excitement in response to novelty, impulsive decision making, and active avoidance of monotony or frustration. It has been suggested that HA is associated with individual variation in serotonin level and is characterized by inhibition, social withdrawal, shyness, and slow adaptation to change. ${ }^{18}$ However, the RD and P dimensions do not directly correspond to any dimensions of the BAS/BIS. This conceptual ambiguity of the $\mathrm{RD}$ and $\mathrm{P}$ dimensions may be related to the known instability and weak internal consistency of these dimensions. ${ }^{19,20}$ The $\mathrm{RD}$ dimension is functionally related to the $\mathrm{C}$ and SD dimensions, and the P dimension appeared to be more closely related to the traits needed to transcend self-limitation against suffering.

Second, the social factor in the traumatized group was interpreted as being concerned with the reinforcement principle: gaining and maintaining positive reward from society by depending on others $(\mathrm{RD})$, identifying others $(\mathrm{C})$, and identifying one's goal (SD). In other words, these dimensions are concerned with the same goal and positive rewards, which can be achieved through the interaction between self and environment. Actually, the collapse of the three dimensions into the same factor agrees with previous findings that show over- lapped factorial loadings of the $\mathrm{RD}$ subscales with those of the $\mathrm{C}^{20}$ and the $\mathrm{SD}$ with the $\mathrm{C}^{21}$ Additionally, high positive intercorrelations among the $\mathrm{RD}, \mathrm{C}$, and $\mathrm{SD}$ have also been reported in prior studies. ${ }^{192,20,22}$

Finally, the subscales that constitute the existential factor of the traumatized sample have been consistently suggested as prerequisites for self-transcendence, the highest level of selfactualization..$^{23}$ In other words, in order to transcend beyond our human limitations such as suffering and mortality, theorists have proposed that we need to accept nothingness and take responsibility for our own existence ${ }^{24}$ It may be that SD4 reflects the ability to accept personal limitations. Self-transcendence also involves exploring new positive meanings within negative experiences ${ }^{24}$ The NS1 reflects a tendency toward exploratory activity in response to novelty. It is also important in self-transcendence to pursuit new possibilities despite failures ${ }^{25}$ The $P$ may reflect a tendency to be industrious, ambitious, overachieving and perseverant despite frustration.

Thus, the factorial allocation of NS1, P, SD4, and all ST subscales into the same factor is not an unexpected random finding but converges with evidence suggesting that NS1 shows relatively complex loadings, and that $\mathrm{P}$ does not load highly on a separate factor. ${ }^{26}$ Moreover, Guietrrez et al. ${ }^{20}$ found the same factorial collapse of these subscales in a joint factor analysis, although it was regarded as a residual factor having no clear psychobiological interpretation. This joint factor analysis of instruments showed a four-factor solution of the TCI including anxiety/inhibition, stimulus-seeking, affiliation, and a fourth residual factor. The three-factor solution in the traumatized sample of the present study corresponds with the result of a previous joint factor analysis which used four personality assessments. ${ }^{20}$

It is also important to note that previous studies, based on normative and clinical samples, consistently reported negative correlations between HA and SD and between NS and HA..$^{8,16,22}$ The non-traumatized sample in the present study also showed similar results. However, for the traumatized sample, all seven 
Table 4. Cronbach alphas of the original seven scales in the traumatized and normative samples

\begin{tabular}{lcc}
\hline \multicolumn{1}{c}{ Dimensions } & $\begin{array}{c}\text { Traumatized } \\
\text { sample }\end{array}$ & $\begin{array}{c}\text { Normative } \\
\text { sample }\end{array}$ \\
\hline Novelty seeking & 0.87 & 0.50 \\
Harm avoidance & 0.90 & 0.68 \\
Reward dependence & 0.80 & 0.43 \\
Persistence & 0.82 & 0.47 \\
Self-directedness & 0.78 & 0.66 \\
Cooperativeness & 0.93 & 0.67 \\
Self-Transcendence & 0.81 & 0.76 \\
\hline
\end{tabular}

scales were highly and positively inter-correlated (Table 3). Therefore, this down-regulation of the personality factor structure in the traumatized sample appeared to be caused by this strengthening of the correlations among personality subscales that share meaningfully related functions as a response to the trauma.

In sum, the functionally meaningful relationship among subscales of the three factors in the traumatized sample indicate that the present three-factor structure of personality is not a random error but may result from the systemic restructuring mechanism of personality in response to trauma.

Analytical causal explanations of the factor model in the traumatized sample are derived from a literature review on the taxonomy of personality. Indeed, the inconsistency of the factor structures of the TCIs between the two samples in this study parallels the long-standing history of the disagreement between various schools of personality regarding the universal taxonomy of personality traits. Some have proposed as few of two or three, and others have proposed as many as five and seven. ${ }^{12,27}$ However, it should be noted that these various taxonomies of personality were developed based on the different samples. That is, the five- and seven-factor models of personality were validated from normative samples, while the tripartite model of existential psychology (i.e., biological-socialpsychological dimensions of Biswagner; body-mind-spirit of Frankl) was based on results from samples of trauma victims. As predicted, the three-factor solution of the present traumatized group is consistent with the tripartite model of personality (i.e., body-mind-spirit), while the five-factor solution of the non-traumatized group corresponds to Cloninger's sevenfactor model. This supports the validity of the present findings and enables us to understand the causal reason of the reorganization of personality factors in response to traumata.

Three limitations may be addressed in the generalization of the present results. First, the small sample size of the traumatized group was small and larger studies are needed to replicate these results. However, for each data set, the values of the Kaiser-Meyer-Olkin index of sampling adequacy ranging from 0 (inadequate) to 1 (excellent), which is a measure of the factorability of the correlation matrices on which the factor analyses were based, were 0.91 in the traumatized group and 0.80 in the non-traumatized group. Further, the internal consistency coefficients of the higher-order TCI dimensions in the traumatized sample were high, ranging from $0.56(\mathrm{RD})$ to $0.94(\mathrm{C})$, and were moderate in the control group, ranging from $0.43(\mathrm{RD})$ to $0.76(\mathrm{ST})$ (Table 4), suggesting that the validity of these results. These results are also consistent with early TCI reports. ${ }^{22}$ Therefore, the correlation matrices of this study were suitable for factor analysis. Second, our traumatized sample was homogeneous in exposure to the type of trauma, age distribution, and time frame assessed. This facet could be both a limitation and a major strength, while the present results are unable to be generalized to other types of trauma, this study overcame the limitations of previous studies that failed to control for the type of traumatic event. Finally, as the TCI was carried in 3 months after the traumatic exposure, structural reorganization of personality system in the traumatized group might not be enduring changes. Furthermore, it could not be ruled out that the structural changes might be affected by the current PTSD symptoms of the victims. Therefore, long-term follow-up studies are needed to examine whether the structural changes of personality were temporal or enduring.

\section{REFERENCES}

1. Caspi A, Roberts BW, Shiner RL. Personality development: stability and change. Ann Rev Psychol 2005;56:453-484.

2. Alexander F. Neurosis and Creativity. Am J Psychoanal 1964;24:116-130.

3. Hirschfeld RM, Klerman GL, Clayton PJ, Keller MB. Personality and depression. Empirical findings. Arch General Psychiatry 1983;40:993998.

4. Finkel NJ. Strens, traumas, and trauma resolution. Am J Community Psychol 1975;3:173-178.

5. Aldwin CM, Sutton KJ, Lachman M. The development of coping resources in adulthood. J Pers 1996;64:837-871.

6. Miller WR. The phenomenon of quantum change. J Clin Psychol 2004; 60:453-460.

7. Tedeschi RG, Calhoun LG. The Posttraumatic Growth Inventory: measuring the positive legacy of trauma. J Trauma Stress 1996;9:455-471.

8. Sato T, Narita T, Hirano S, Kusunoki K, Goto M, Sakado K, et al. Factor validity of the temperament and character inventory in patients with major depression. Compr Psychiatry 2001;42:337-341.

9. Hur JW, Kim YK. Comparison of clinical features and personality dimensions between patients with major depressive disorder and normal control. Psychiatry Investig 2009;6:150-155.

10. Kim KI, Won HT, Lee JH, Kim KY. Standadization study of Symptom Check List-90 in Korea I: characteristics of normal responses. J Korean Neuropsychiatr Assoc 1978;17:449-458.

11. Sung SM, Kim JH, Yang E, Abrams KY, Lyoo IK. Reliability and validity of the Korean version of the Temperament and Character Inventory. Compr Psychiatry 2002;43:235-243.

12. Cloninger CR, Svrakic DM, Przybeck TR. A psychobiological model of temperament and character. Arch Gen Psychiatry 1993;50:975-990.

13. Lukas E. [Logotherapy. Victor Frankl 1905-1997]. Wien Klin Wochen- 
schr 1998;110:275-278.

14. Gray JA. The psychophysiological basis of introversion-extraversion. Behav Res Ther 1970;8:249-266.

15. Gray JA. Brain systems that mediate both emotion and cognition. Cognition Emotion 1990;4:269-288.

16. Stellar E. The physiology of motivation. 1954. Psychol Rev 1994;101: 301-311.

17. Carver CS, White TL. Behavioral inhibition, behavioral activation and affective responses to impending reward and punishment: The BIS/ BAS scales. J Pers Soc Psychol 1994;67:319-333.

18. Cloninger CR. A systematic method for clinical description and classification of personality variants. A proposal. Arch Gen Psychiatry 1987; 44:573-588.

19. Cloninger CR, Przybeck TR, Svrakic DM. The Tridimensional Personality Questionnaire: U.S. normative data. Psychol Rep 1991;69:10471057.

20. Gutierrez F, Torrens M, Boget T, Martin-Santos R, Sangorrin J, Perez G, et al. Psychometric properties of the Temperament and Character In- ventory (TCI) questionnaire in a Spanish psychiatric population. Acta Psychiatr Scand 2001;103:143-147.

21. de la Rie SM, Duijsens IJ, Cloninger CR. Temperament, character, and personality disorders. J Pers Disord 1998;12:362-372.

22. Cloninger CR. Temperament and personality. Curr Opin Neurobiol 1994;4:266-273

23. Maslow A. A theory of human motivation. Psychol Rev 1943;50:370396.

24. Frankl VE. The feeling of meaninglessness: a challenge to psychotherapy. Am J Psychoanal 1972;32:85-89.

25. Frankl VE. Logotherapy and existential analysis--a review. Am J Psychother 1966;20:252-260.

26. Stallings MC, Hewitt JK, Cloninger CR, Heath AC, Eaves LJ. Genetic and environmental structure of the Tridimensional Personality Questionnaire: three or four temperament dimensions? J Pers Soc Psychol 1996;70:127-140.

27. Goldberg LR. An alternative "description of personality": the big-five factor structure. J Pers Soc Psychol 1990;59:1216-1229. 